\title{
Composición e invarianza factorial del Autoinforme de Barreras para la Práctica de Ejercicio Físico (ABPEF-M) en universitarios mexicanos deportistas
}

\section{Composition and factorial invariance of the Self Report Barriers for the Practice of Physical Activity Scale (ABPEF-M) in Mexican university athletes}

\section{Estrutura e invariancia fatorial do Autoinforme de Barreiras pala Prática de Exercício Físico (ABPEF-M) em universitários atletas mexicanos}

\author{
Mondaca, F.1, Zueck, M. C.2, Mayorga-Vega, D.2, Flores, L. A.1, Benitez, Z. P.1, Peinado, J. E.1 \\ 1Facultad de Ciencias de la Cultura Física, Universidad Autónoma de Chihuahua, México; \\ ${ }_{2}$ Departamento de Didáctica de la Expresión Musical, Plástica y Corporal, Universidad de Jaén
}

\begin{abstract}
RESUMEN
El presente estudio analiza las propiedades psicométricas propuestos por Rodríguez-Villalobos, Viciana, Tristán y Blanco para el Autoinforme de Barreras para la Práctica de Ejercicio Físico (ABPEF-M) en hombres y mujeres deportistas universitarios. La muestra total fue de 413 universitarios mexicanos deportistas 174 mujeres y 239 hombres, con edades comprendidas entre $18-26$ años $(M=20.76$; $D E=2.06)$. La estructura factorial del cuestionario se analizó mediante análisis factoriales confirmatorios. Los análisis, muestran que una estructura tetrafactorial (imagen corporal, fatiga, obligaciones y ambiente) es viable y adecuada tanto para la muestra total $(G F I=.951$; $R M S E A=.071 ; C F I=.972)$ como para las poblaciones de hombres $(G F I=.944 ; R M S E A=.067)$ y mujeres $(G F I$ $=.917 ; R M S E A=.081)$. La estructura de cuatro factores, atendiendo a criterios estadísticos y sustantivos, ha mostrado adecuados indicadores de ajuste de fiabilidad y validez. Además, la estructura factorial, las cargas factoriales y los interceptos se consideran invariantes de acuerdo al género; sin embargo, no se encontraron diferencias entre hombres y mujeres deportistas en cuanto su percepción sobre la presencia de barreras en las cuatro dimensiones estudiadas. Palabras clave: validez; fiabilidad; psicometría; evaluación psicológica.
\end{abstract}




\title{
Mondaca, Zueck, Mayorga-Vega, Flores, Benitez, Peinado
}

\begin{abstract}
The aim of this research was to analyze the psychometric properties proposed by Rodríguez- Villalobos, Viciana, Tristán and Blanco for the Barriers for the Practice of Physical Activity Scale (ABPEF-M) in university athletes. The total sample has been composed by 413 Mexican university athletes, 174 women and 239 men; participants' age ranged from $18-26$ years $(M=20.76, S D=2.06)$. The factor structure of the questionnaire was analyzed through confirmatory factor analysis. The analysis showed a viable and adequate tetrafactorial structure (body image, fatigue, obligations, and environment $)$ for the total sample $(G F I=.951 ; R M S E A=.071 ; C F I=.972)$ and the populations of men $(G F I=.944 ; R M S E A=.067)$ and women $(G F I=.917 ; R M S E A=.081)$. The four factor structure, according to statistical and substantive criteria, showed adequate indicators of reliability and validity adjustment. On the other hand, the factorial structure, factorial loads and the intercepts are considered invariant by gender; however, no differences were found between male and female athletes with regards to their perception of the presence of barriers in the four dimensions studied.
\end{abstract}

Keywords: validity; reliability; psychometry; psychological assessment.

\section{RESUMO}

O objetivo deste estudo foi examinar a estructura e invariância de gênero Auto-relato de Barreiras à Prática de Exercício Físico (ABPEF-M) proposto por Rodríguez- Villalobos, Viciana, Tristán e Blanco em atletas universitários mexicanos. A amostra total foi de 413 atletas mexicanos universitários, 174 mulheres e 239 homens, com idades entre 18-26 anos $(\mathrm{M}=20.76$; $\mathrm{DP}=2.06)$. A estrutura fatorial do questionário foi analisada por análises fatoriais confirmatórias. Os resultados mostraram que uma estrutura de quatro fatores (imagem corporal/ ansiedade social físico, fadiga/ preguiça, obrigações/ falta de tempo, e meio ambiente/ instalações) é viável e adequado tanto para a amostra total $(\mathrm{GFI}=.951 ; \mathrm{RMSEA}=.071 ; \mathrm{CFI}=.972) \mathrm{e}$ para as populações de homens $(\mathrm{GFI}=.944 ; \mathrm{RMSEA}=.067)$ e mulheres $(\mathrm{GFI}=.917$; RMSEA $=.081)$. Com base em critérios estatísticos e substantivos, a estrutura de quatro fatores mostrou adequada confiabilidade e validade dos indicadores de ajuste. Além disso, a estrutura fatorial, as cargas fatoriais e os interceptos eram invariantes de acordo com o gênero. Não foram encontradas diferenças entre atletas homens e mulheres na sua percepção da presença de barreiras nas quatro dimensões estudadas.

Palavras chave: validade; confiabilidade; psicometria; avaliação psicológica.

\section{INTRODUCCIÓN}

Existe una fuerte evidencia científica de que la práctica habitual de actividad física (AF) supone numerosos beneficios sobre la salud y el bienestar de las personas (Murgui, García y García, 2016; Poitras et al., 2016; Requena-Pérez, Martín-Cuadrado y LagoMarín, 2015; Urrutia, Azpillaga, de Cos y Muñoz, 2010). Por ejemplo, en adultos la práctica regular de AF está asociado con un menor riesgo de enfermedad cardiovascular (Li y Siegrist, 2012), hipertensión (Huai et al., 2013), diabetes (Sigal, Kenny, Wasserman, Castaneda-Sceppa y White, 2006) y cáncer (Zhong et al., 2016). Lamentablemente, hoy en día en todo el mundo alrededor del $28 \%$ de los adultos no tienen hábitos saludables de $\mathrm{AF}$, siendo aún peor en regiones como América Latina (39\%) (Guthold, Stevens, Riley y Bull, 2018; Samperio, JiménezCastuera, Lobato, Leyton y Claver, 2016). Además, existe una fuerte brecha de género, habiendo un mayor porcentaje de mujeres físicamente inactivas que de hombres (por ejemplo, $8 \%$ en todo el mundo y $9 \%$ en América Latina) (Guthold et al., 2018).

Reconociendo el fuerte vínculo entre los hábitos de $\mathrm{AF}$ y la salud, unido con los altos índices de inactividad física, actualmente una prioridad de las políticas de salud pública es promocionar en la población hábitos saludables de AF (World Health Organization, 2018). Por ejemplo, los estados miembros de la Organización Mundial de la Salud acordaron una reducción relativa del $10 \%$ en la prevalencia de la inactividad física para 2025 (World Health Organization, 2014) y del 15\% para el 2030 (World Health Organization, 2018). Entre múltiples factores psicológicos, existe evidencia científica de que las barreras hacia el ejercicio físico están negativamente asociadas con los niveles 


\section{Composición e Invarianza factorial del cuestionario ABPEF-M}

habituales de AF (Gunnell, Brunet, Wing y Bélanger, 2015). Por ello, las principales políticas de promoción de salud pública se centran en acciones dirigidas a la reducción de barreras hacia la AF (World Health Organization, 2018).

Por tanto, las barreras percibidas por la población para la realización de AF adquieren enorme importancia para incrementar sus niveles habituales de AF. La literatura previa ha mostrado que aspectos como la ansiedad física social ligada a la imagen corporal, la fatiga o pereza, la falta de tiempo por obligaciones, o el ambiente y falta de instalaciones, son identificadas como los principales motivos de la inactividad física (Niñerola, Capdevila y Pintanel, 2006). En cuanto a los instrumentos de medida de estas barreras hacia la $\mathrm{AF}$, la aportación en lengua española más importante es el Autoinforme de Barreras para la Práctica de Ejercicio Físico (ABPEF) (Niñerola et al., 2006). Aunque el ABPEF fue originalmente diseñado por (Capdevila, 2005) con 20 ítems, posteriormente Niñerola et al. (2006) identificaron un instrumento de 17 ítems con propiedades psicométricas adecuadas, el cual estaba compuesto por las siguientes cuatro barreras: (1) Imagen corporal/ Ansiedad física social, relacionadas con la ansiedad física y la preocupación sobre cómo nos ven los demás durante la práctica de AF; (2) Fatiga/ Pereza, relacionadas con los motivos personales tales cansancio, pereza o falta de voluntad para hacer AF; (3) Obligaciones/ Falta de tiempo, relacionadas con la falta de tiempo para la práctica de $\mathrm{AF}$, y (4) Ambiente/ Instalaciones, relacionadas con las limitaciones por el ambiente e instalaciones disponibles para la realización de AF.

Es de suma importancia comprobar la estructura factorial de un instrumento y la equivalencia psicométrica del mismo en distintos grupos poblacionales (Abalo, Lévy, Rial y Varela, 2006). En este sentido, recientemente Rodríguez-Villalobos et al. (2017) examinaron las propiedades psicométricas del ABPEF en estudiantes universitarios mexicanos. Aunque los resultados mostraron que la estructura de cuatro factores con 17 ítems era fiable y válida, en la población mexicana el instrumento era significativamente mejor cuando se reducía a 13 ítems (ABPEF-M). Por otro lado, estos autores encontraron que la estructura factorial, las cargas factoriales y los interceptos eran invariantes entre deportistas y no deportistas. Sin embargo, a pesar de la vital importancia del análisis de las diferencias en las barreras para la práctica de $\mathrm{AF}$ entre hombres y mujeres (Colmenares-Pedraza y Herrera-Medina, 2018; Rodríguez-Castellanos, Valencia, GaitánGuzmán, González-Rodríguez y León-Díaz, 2017; Sevil, Práxedes, Zaragoza, Del Villar y GarcíaGonzález, 2017), Rodríguez-Villalobos et al. (2017) no examinaron la invarianza factorial del ABPEF-M por género. Comprender las posibles diferencias por género de todos estos factores permitirá mejorar la efectividad de los programas de promoción de hábitos de AF, mejorando así los niveles de salud y calidad de vida de la población. No obstante, previamente se debe comprobar que los instrumentos utilizados son invariantes por género, por lo que, el objetivo de la presente investigación instrumental (Ato, López y Benavente, 2013) fue examinar la composición e invarianza factorial por género del ABPEF-M propuesta por Rodríguez-Villalobos et al. (2017) en deportistas universitarios mexicanos.

\section{MATERIAL Y MÉTODOS}

\section{Participantes}

La muestra se conformó por 413 estudiantes universitarios, 174 mujeres y 239 hombres, de La Paz Baja California Sur México que practicaban algún deporte y participan regularmente en torneos y/o competencias deportivas. Esta se obtuvo mediante un muestreo por conveniencia. La edad de los participantes fluctuó entre los 18 y 26 años, con una media de 20.76 años y una desviación estándar de 2.06 años. Todos los participantes cumplimentaron el cuestionario voluntariamente.

\section{Instrumentos}

Se utilizó el Autoinforme de Barreras para la Práctica de Ejercicio Físico (ABPEF-M) de RodríguezVillalobos et al. (2017), el cual es una versión adaptada, de la versión original del ABPEF de Niñerola et al. (2006). Consta de 13 ítems que se agrupan en las siguiente cuatro dimensiones: imagen corporal/ ansiedad físico social (5 ítems; por ejemplo., "Sentir incomodidad por el aspecto que tengo con ropa deportiva"); fatiga/ pereza (3 ítems; por ejemplo, "Notar cansancio o fatiga de forma habitual a lo largo del día"); obligaciones/ falta de tiempo (3 ítems; por ejemplo, "Tener demasiado trabajo", y ambiente/ instalaciones ( 2 ítems; por ejemplo, "Encontrarme a disgusto con la gente que hace ejercicio conmigo"). 


\section{Mondaca, Zueck, Mayorga-Vega, Flores, Benitez, Peinado}

Los participantes respondieron de acuerdo a una escala tipo Likert de 0 a 10 puntos, donde valores cercanos a 0 indican "una razón poco probable que me impide practicar ejercicio físico las próximas semanas", y valores cercanos a 10 indican una "razón muy probable que me impide practicar ejercicio físico". El ABPEF-M de Rodríguez-Villalobos et al. (2017) ha mostrado propiedades psicométricas adecuadas en estudiantes universitarios mexicanos $(G F I=.961 ; R M S E A=.065 ; T L I=.951 ; C F I=.969)$.

\section{Procedimiento}

Se invitó a participar en el estudio a todos los estudiantes universitarios de la ciudad de La Paz Baja California Sur México que practicaban algún deporte y participaban regularmente en torneos y/o competencias deportivas. Los estudiantes que aceptaron participar firmaron el consentimiento informado. Luego se aplicó el cuestionario ABPEF-M de Rodríguez-Villalobos et al. (2017) a través de medios informáticos en las propias aulas de los centros educativos en una sesión de aproximadamente 30 minutos. Al inicio de cada sesión se hizo una pequeña introducción sobre los objetivos y la importancia de la investigación, así de cómo contestar el instrumento. Se les pidió fueran lo más honestos posible y se les garantizó la confidencialidad de los datos que se obtuvieran. Las instrucciones de cómo responder se encontraban en las primeras pantallas, antes del primer ítem del instrumento. Al término de la sesión se les agradeció su participación. Una vez aplicado el instrumento se procedió a recopilar y a analizar los datos mediante el paquete SPSS 18.0 y AMOS 21.0.

\section{Análisis estadístico}

Con el fin de examinar la composición e invarianza factorial por género del ABPEF-M propuesta por Rodríguez-Villalobos et al. (2017) en deportistas universitarios mexicanos, el análisis psicométrico se realizó en dos etapas: (1) Análisis de las propiedades psicométricas del instrumento, y (2) Análisis de invarianza factorial.

Análisis de las propiedades psicométricas del instrumento. El primer paso del análisis de las propiedades psicométricas del cuestionario consistió en calcular la asimetría y la curtosis de cada ítem, para determinar si se cumplía el supuesto de normalidad. Luego se sometieron a comparación dos modelos de medida: el ABPEF-M1A, que respondía a una estructura tetrafactorial acorde a la distribución original de los ítems dentro del cuestionario según Rodríguez-Villalobos et al. (2017) y el ABPEF-M1B, que correspondía a la estructura factorial del modelo anterior sin los ítems que de acuerdo a los índices de modificación resultaron no adecuados. Posteriormente, se calculó la fiabilidad de los factores del mejor de los modelos de medida obtenido a través del Coeficiente de Alfa de Cronbach (Elosua y Zumbo, 2008; Nunnally y Bernstein, 1995) y Coeficiente Omega (Revelle y Zinbarg, 2009; Sijtsma, 2009).

La validez convergente se estimó a través de la significatividad y tamaño de las saturaciones de los ítems sobre cada factor y de la varianza media extraída (AVE) que de acuerdo a Fornell y Larcker (1981) debe ser mayor a .50. Mientras que para estimar la validez discriminante se utilizó el test de varianza extraída propuesto también por Fornell y Larcker (1981) donde se calcula la AVE para cada uno de los factores del modelo y se compara con el cuadrado de la correlación entre los factores, luego si las AVE de los dos factores superan el cuadrado de la correlación entre ellos se puede afirmar que hay validez discriminante.

Análisis de invarianza factorial. Con el fin de obtener una prueba que presente las mejores propiedades para la conformación de los puntajes del cuestionario ABPEF-M en mujeres y hombres se llevó a cabo un análisis de la invarianza factorial, tomando como base el mejor modelo obtenido en la muestra total (es decir, modelo ABPEF-M1B). Luego se calculó la fiabilidad, en ambas muestras, de cada una de las dimensiones a través del Alfa de Cronbach y el Coeficiente Omega (Revelle y Zinbarg, 2009).

Todos los análisis factoriales confirmatorios se realizaron mediante el software AMOS 21 (Arbuckle, 2012). Las varianzas de los términos de error fueron especificadas como parámetros libres y en cada una de las variables latentes (factores) se fijó uno de sus coeficientes estructurales a uno, para que su escala fuera igual a la de una de las variables observables (ítems). El método de estimación empleado fue el de Máxima Verosimilitud (ML) con la aplicación de procedimientos de remuestreo bootstrap para los casos de no normalidad (Byrne, 2016). En AMOS 21.0 el ML es especialmente robusto para posibles casos de no normalidad, especialmente si la muestra es suficientemente amplia y los valores de asimetría y curtosis no son extremos (asimetría $<|2|$ y curtosis $<$ 


\section{Composición e Invarianza factorial del cuestionario ABPEF-M}

|7|). Además, siguiendo la recomendación de Thompson (2004), cuando se emplea el análisis factorial confirmatorio se debe corroborar no sólo el ajuste de un modelo teórico, sino que es recomendable comparar los índices de ajuste de varios modelos alternativos para seleccionar el mejor.

Para evaluar el ajuste del modelo se emplearon el estadístico Chi-cuadrado, el índice de bondad de ajuste (GFI) y el error cuadrático medio de aproximación (RMSEA) como medidas absolutas de ajuste. El índice de bondad ajustado ( $A G F I$ ), el Índice Tucker-Lewis (TLI) y el índice de bondad de ajuste comparativo ( $C F I)$ como medidas de ajuste incremental. La razón de Chi-cuadrado sobre los grados de libertad $(C M I N / G L)$ y el Criterio de Información de Akaike
(AIC) como medidas de ajuste de parsimonia (Byrne, 2016; Gelabert et al., 2011).

\section{RESULTADOS}

\section{Asimetría y curtosis de los ítems del cuestionario}

En la Tabla 1, además de las medias y desviaciones estándar, se resumen los valores de asimetría y curtosis de las distintas variables contempladas en el modelo de medida. La mayoría de las variables muestran valores de asimetría de \pm 2.50 y \pm 3.80 de curtosis, por lo que se infiere que las variables se ajustan razonablemente a una distribución normal (normalidad univariada); sin embargo, el índice multivariado de Mardia por encima del valor 70 indica ausencia de normalidad multivariada (Rodríguez y Ruiz, 2008).

Tabla 1

Medias, desviaciones estándar, asimetría y curtosis de los ítems del cuestionario ABPEF-M. Muestra total.

\begin{tabular}{lcccc}
\multicolumn{1}{c}{ Ítem } & M & DE & AS & CU \\
\hline 1. Sentir incomodidad por el aspecto que tengo con ropa deportiva & 1.20 & 2.39 & 2.10 & 3.41 \\
2. Tener demasiado trabajo & 3.29 & 3.13 & 0.59 & -0.82 \\
3. Sentir que mi aspecto físico es peor que el de los demás & 0.97 & 2.17 & 2.65 & 6.61 \\
4. Tener demasiadas obligaciones familiares & 2.12 & 2.70 & 1.24 & 0.69 \\
5. No estar "en forma" para practicar ejercicio & 1.24 & 2.34 & 1.91 & 2.93 \\
6. Falta de voluntad para ser constante & 1.56 & 2.41 & 1.59 & 1.69 \\
7. Pensar que la otra gente está en mejor forma que yo & 1.13 & 2.22 & 2.20 & 4.33 \\
8. No encontrar el tiempo necesario para el ejercicio & 2.11 & 2.76 & 1.18 & 0.33 \\
9. Notar cansancio o fatiga de forma habitual a lo largo del día & 1.91 & 2.47 & 1.33 & 1.04 \\
10. Pensar que los demás juzgan mi apariencia física & 0.91 & 2.13 & 2.67 & 6.63 \\
11. Encontrarme a disgusto con la gente que hace ejercicio conmigo & 1.17 & 2.32 & 2.12 & 3.80 \\
12. Sentir vergüenza porque me están mirando mientras hago ejercicio & 0.97 & 2.18 & 2.36 & 4.63 \\
13. Las instalaciones o los entrenadores no sean adecuados & 1.57 & 2.60 & 1.66 & 1.79 \\
& \multicolumn{1}{c}{ índice multivariado de Mardia } & & &
\end{tabular}

Nota: $\mathrm{M}=$ media, $\mathrm{DE}=$ desviación estándar, $\mathrm{AS}=$ asimetría; $\mathrm{CU}$ = curtosis

\section{Análisis factorial confirmatorio muestra total}

Los resultados globales del análisis factorial confirmatorio para el modelo ABPEF-M1A, el cual corresponde a una estructura tetrafactorial acorde a la distribución original de los ítems dentro del cuestionario, indicaron que el modelo de medición es apenas aceptable $(G F I=.911 ; R M S E A=.092 ; C F I$ $=.942)$ (Tabla 2).

Cuadernos de Psicología del Deporte, 20, 2 (mayo)
Los factores del modelo ABPEF-M1A explicaron aproximadamente el 76\% de la varianza y 11 de los 13 ítems saturaron por encima de .70 en su dimensión prevista (Tabla 3). Por otro lado, con la excepción de las correlaciones entre el factor 2 con los factores 1 y 3 , se observaron intercorrelaciones moderadas entre los factores, además de que la mayoría de las AVE (Tabla 4) superan el cuadrado de la correlación entre ellos, lo que sugiere la existencia de validez discriminante. 


\section{Mondaca, Zueck, Mayorga-Vega, Flores, Benitez, Peinado}

Los resultados globales del análisis factorial confirmatorio del segundo modelo sometido a prueba (ABPEF-M1B), el cual correspondía a la estructura factorial del modelo anterior (ABPEF-M1A) sin los ítems 5 y 12, indicaron que este modelo de medición era mejor que el modelo anterior y que su ajuste fue óptimo $(G F I=.951 ; R M S E A=.071 ; C F I=.972)$ (Tabla 2). Los factores de este modelo explicaron aproximadamente el $79 \%$ de la varianza. Por otro lado, todos los ítems (con excepción del ítem 13) saturan igual o por encima de .70 en su dimensión prevista (Tabla 3). Observándose intercorrelaciones moderadas entre los factores, además de que todas las AVE (Tabla 4) superan el cuadrado de la correlación entre ellos, lo que sugiere la existencia de validez discriminante.

Tabla 2

Índices absolutos, incrementales y de parsimonia para los modelos generados.

\begin{tabular}{|c|c|c|c|c|c|c|c|c|}
\hline \multirow[b]{2}{*}{ Modelo } & \multicolumn{3}{|c|}{ Índices absolutos } & \multicolumn{3}{|c|}{ Índices incrementales } & \multicolumn{2}{|c|}{ Índices de parsimonia } \\
\hline & $\chi^{2}$ & GFI & RMSEA & AGFI & TLI & CFI & CMIN/DF & AIC \\
\hline ABPEF-M1A & $259.911 *$ & .911 & .092 & .860 & .922 & .942 & 4.481 & 325.911 \\
\hline ABPEF-M1B & $113.226^{*}$ & .951 & .071 & .913 & .959 & .972 & 3.060 & 171.226 \\
\hline
\end{tabular}

Nota: $*$ p <.05; GFI = índice de bondad de ajuste; RMSEA = raíz del error medio; AGFI = índice corregido de la bondad de ajuste; TLI = índice de Tucker-Lewis; $\mathrm{CFI}=$ índice de ajuste comparativo; CMIN/DF = índice de ajuste chi cuadrado dividido por los grados de libertad; $\mathrm{AIC}=$ criterio de información de Akaike

Tabla 3

Soluciones estandarizadas análisis factorial confirmatorio para los Modelos ABPEF-MIA y ABPEF-MIB.

\begin{tabular}{|c|c|c|c|c|c|c|c|c|c|}
\hline \multirow[b]{2}{*}{ Ítem } & \multicolumn{5}{|c|}{ ABPEF-M1A } & \multicolumn{4}{|c|}{ ABPEF-M1B } \\
\hline & & $\mathrm{F} 1$ & $\mathrm{~F} 2$ & F3 & F4 & $\mathrm{F} 1$ & F2 & F3 & F4 \\
\hline \multicolumn{10}{|c|}{ Pesos Factoriales } \\
\hline Ítem No. 1 & & .73 & & & & .74 & & & \\
\hline Ítem No. 3 & & .78 & & & & .80 & & & \\
\hline Ítem No. 7 & & .86 & & & & .87 & & & \\
\hline Ítem No. 10 & & .87 & & & & .87 & & & \\
\hline Ítem No. 12 & & .78 & & & & - & & & \\
\hline Ítem No. 5 & & & .72 & & & & - & & \\
\hline Ítem No. 6 & & & .73 & & & & .76 & & \\
\hline Ítem No. 9 & & & .74 & & & & .78 & & \\
\hline Ítem No. 2 & \multicolumn{5}{|c|}{.68} & & \multicolumn{3}{|c|}{.70} \\
\hline Ítem No. 4 & \multicolumn{5}{|c|}{.76} & & \multicolumn{3}{|c|}{.74} \\
\hline Ítem No. 8 & \multicolumn{5}{|c|}{.83} & & \multicolumn{3}{|c|}{.85} \\
\hline Ítem No. 11 & & & & & .87 & & & & .86 \\
\hline Ítem No. 13 & & & & & .64 & & & & .65 \\
\hline \multicolumn{10}{|c|}{ Correlaciones Factoriales } \\
\hline & $\mathrm{F} 1$ & - & & & & - & & & \\
\hline & F2 & .94 & - & & & .85 & - & & \\
\hline & F3 & .63 & .93 & - & & .62 & .90 & - & \\
\hline & $\mathrm{F} 4$ & .86 & .88 & .60 & - & .82 & .86 & .60 & - \\
\hline
\end{tabular}

Nota: F1 = Imagen Corporal / Ansiedad física social F2 = Fatiga / Pereza F3 = Obligaciones $/$ Falta de tiempo F4 = Ambiente / Instalaciones

Los factores obtenidos en los análisis factoriales confirmatorios, en ambos modelos, alcanzan valores de consistencia interna por encima de .70; evidenciando una consistencia interna adecuada para 


\section{Composición e Invarianza factorial del cuestionario ABPEF-M}

este tipo de subescalas, sobre todo si se tiene en cuenta el número reducido de ítems en cada uno de los factores (Tabla 4). ). En cuanto a la varianza media extraída todos los factores obtienen valores por encima de .50 .

Tabla 4

Coeficiente omega y alfa para los factores obtenidos en los Modelos ABPEF-MIA y ABPEF-MIB.

\begin{tabular}{|c|c|c|c|c|c|c|}
\hline \multirow[b]{2}{*}{ Factor } & \multicolumn{3}{|c|}{ ABPEF-M1A } & \multicolumn{3}{|c|}{ ABPEF-M1B } \\
\hline & $\Omega(\mathrm{FC})$ & $\alpha$ & AVE & $\Omega(\mathrm{FC})$ & $\alpha$ & AVE \\
\hline Imagen Corporal / Ansiedad física social & .902 & .907 & .649 & .892 & .901 & .675 \\
\hline Fatiga / Pereza & .774 & .775 & .533 & .744 & .750 & .593 \\
\hline Obligaciones / Falta de tiempo & .802 & .798 & .573 & .809 & .798 & .586 \\
\hline Ambiente / Instalaciones & .732 & .713 & .583 & .731 & .713 & .585 \\
\hline
\end{tabular}

Nota $:$ FC $=$ fiabilidad compuesta $\mathrm{AVE}=$ varianza media extraída

\section{Análisis factoriales confirmatorios hombres y mujeres}

Tanto en la muestra de mujeres como de hombres todas las variables mostraron valores de asimetría de \pm 2.50 y \pm 5.00 de curtosis. Sin embargo, el índice multivariado de Mardia por encima del valor 70 indicó ausencia de normalidad multivariada (Rodríguez y Ruiz, 2008).

De acuerdo a los resultados de la Tabla 5 el análisis factorial confirmatorio de 11 ítems agrupados en cuatro factores en la muestra de mujeres fue aceptable
$(G F I=.917 ; R M S E A=.081)$. Además, de acuerdo a las medidas de ajuste incremental y de parsimonia fue significativamente superior al modelo independiente y muy similar al modelo saturado.

Por otro lado, el análisis factorial confirmatorio en la muestra de hombres (Tabla 5), indicó nuevamente que el modelo de medición tetrafactorial era aceptable $(G F I=.944 ;$ RMSEA $=.067)$. De acuerdo a las medidas de ajuste incremental y de parsimonia fue significativamente superior al modelo independiente y muy similar al modelo saturado.

Tabla 5

Índices absolutos, incrementales y de parsimonia para los modelos generados. Análisis factorial confirmatoriopara mujeres y hombres.

\begin{tabular}{|c|c|c|c|c|c|c|c|c|}
\hline \multirow[b]{2}{*}{ Modelo } & \multicolumn{3}{|c|}{ Índices absolutos } & \multicolumn{3}{|c|}{ Índices incrementales } & \multicolumn{2}{|c|}{ Índices de parsimonia } \\
\hline & $\chi^{2}$ & GFI & RMSEA & AGFI & TLI & CFI & CMIN/DF & AIC \\
\hline \multicolumn{9}{|c|}{ Solución factorial para los mujeres } \\
\hline 11 ítems 4 factores & $89.750^{*}$ & .917 & .081 & .852 & .924 & .949 & 2.426 & 147.750 \\
\hline Saturado & 0.000 & 1.000 & & & & 1.000 & & 132.000 \\
\hline Independiente & $1083.336^{*}$ & .306 & .329 & .167 & .000 & .000 & 19.697 & 1105.336 \\
\hline \multicolumn{9}{|c|}{ Solución factorial para los hombres } \\
\hline 11 ítems 4 factores & $76.091 *$ & .944 & .067 & .900 & .968 & .978 & 2.057 & 134.091 \\
\hline Saturado & 0.000 & 1.000 & & & & 1.000 & & 132.000 \\
\hline Independiente & $1872.380 *$ & .244 & .373 & .093 & .000 & .000 & 34.043 & 1894.380 \\
\hline
\end{tabular}

Nota: $* \mathrm{p}<.05 ; \mathrm{GFI}=$ índice de bondad de ajuste; RMSEA = raíz del error medio; AGFI = índice corregido de la bondad de ajuste; TLI = índice de Tucker-Lewis; CFI = índice de ajuste comparativo; CMIN/DF = índice de ajuste chi cuadrado dividido por los grados de libertad; AIC = criterio de información de Akaike 


\section{Mondaca, Zueck, Mayorga-Vega, Flores, Benitez, Peinado}

De acuerdo a los resultados de la Tabla 6, en ambas muestras la mayoría de los ítems saturaron por encima de .70 en su dimensión (factor) prevista. Observándose además en general intercorrelaciones moderadas entre los factores, además de que la mayoría de las AVE (Tabla 8) superan el cuadrado de la correlación entre ellos, lo que sugiere la existencia de validez discriminante.

Tabla 6

Soluciones estandarizadas para el análisis factorial confirmatorio en ambas muestras.

\begin{tabular}{|c|c|c|c|c|c|c|c|c|}
\hline & \multicolumn{4}{|c|}{ Mujeres } & \multicolumn{4}{|c|}{ Hombres } \\
\hline Ítem & F1 & $\mathrm{F} 2$ & F3 & $\mathrm{F} 4$ & $\mathrm{~F} 1$ & $\mathrm{~F} 2$ & F3 & F4 \\
\hline \multicolumn{9}{|c|}{ Pesos Factoriales } \\
\hline 1. Sentir incomodidad por el aspecto que tengo con ropa deportiva & .71 & & & & .77 & & & \\
\hline 3. Sentir que mi aspecto físico es peor que el de los demás & .81 & & & & .79 & & & \\
\hline 7. Pensar que la otra gente está en mejor forma que yo & .83 & & & & .90 & & & \\
\hline 10. Pensar que los demás juzgan mi apariencia física & .84 & & & & .90 & & & \\
\hline 6. Falta de voluntad para ser constante & & .79 & & & & .74 & & \\
\hline 9. Notar cansancio o fatiga de forma habitual a lo largo del día & & .83 & & & & .76 & & \\
\hline 2. Tener demasiado trabajo & & & 67 & & & & 69 & \\
\hline 4. Tener demasiadas obligaciones familiares & & & .74 & & & & .75 & \\
\hline 8. No encontrar el tiempo necesario para el ejercicio & & & .87 & & & & .84 & \\
\hline 11. Encontrarme a disgusto con la gente que hace ejercicio conmigo & & & & .88 & & & & .85 \\
\hline 13. Las instalaciones o los entrenadores no sean adecuados & & & & .57 & & & & .71 \\
\hline \multicolumn{9}{|c|}{ Correlaciones Factoriales } \\
\hline $\mathrm{F} 1$ & - & & & & - & & & \\
\hline $\mathrm{F} 2$ & .75 & - & & & .93 & - & & \\
\hline F3 & .61 & .82 & - & & .65 & .92 & - & \\
\hline F4 & .70 & .80 & .53 & - & .93 & .91 & .66 & - \\
\hline
\end{tabular}

Nota: F1 = Imagen Corporal / Ansiedad física social F2 = Fatiga / Pereza F3 = Obligaciones / Falta de tiempo F4 = Ambiente / Instalaciones

\section{Invarianza de la estructura factorial entre mujeres y hombres}

Los índices de ajuste obtenidos (Tabla 7) permitieron aceptar la equivalencia de los modelos de medida básicos entre las dos muestras. Aunque el valor de Chi-cuadrado excede al exigido para aceptar la hipótesis de invarianza, el resto de índices contradijeron esta conclusión por lo que nos permite aceptar el modelo base de la invarianza (modelo sin restricciones).

Añadiendo al modelo base restricciones sobre las cargas factoriales caracterizamos la invarianza métrica. Los valores que se recogen en la Tabla 7 permitieron aceptar este nivel de invarianza. El índice de ajuste general (GFI $=.928)$ y el error cuadrático medio de aproximación $($ RMSEA $=.054)$ siguieron aportando información convergente en el sentido de la invarianza métrica. Además, el criterio de información de Akaike (278.713) y el índice comparativo de Bentler (.966) no sufrieron grandes variaciones respecto al modelo anterior. De acuerdo con el criterio para la evaluación de los modelos anidados propuesto por Cheung y Rensvold (2002), quiénes sugieren que si el cálculo de la diferencia de los CFI de ambos modelos anidados disminuye en .01 o menos se debe de dar por bueno el modelo restringido y consecuentemente el cumplimiento de la invarianza factorial, la diferencia entre CFIs obtenida permitió aceptar el modelo de invarianza métrica. Por tanto, se pudo considerar que las cargas factoriales fueron equivalentes en las dos muestras.

Una vez demostrada la invarianza métrica entre las muestras, se evaluó la equivalencia entre interceptos (invarianza factorial fuerte). Los índices (Tabla 7) mostraron un ajuste aceptable de este modelo tanto evaluado de modo independiente como analizándolo respecto a su anidamiento con el modelo de invarianza métrica. La diferencia entre los índices comparativos de Bentler fue de ocho milésimas, el índice de ajuste general de .916 y el error cuadrático medio de aproximación de .056. Aceptada la invarianza fuerte, los dos modelos evaluados fueron equivalentes respecto a los coeficientes factoriales y a los interceptos. 


\section{Composición e Invarianza factorial del cuestionario ABPEF-M}

Tabla 7

Índices de bondad de ajuste de cada uno de los modelos puestos a prueba en la invarianza factorial.

\begin{tabular}{lcccccccc}
\hline \multicolumn{1}{c}{ Modelo } & \multicolumn{7}{c}{ Índice de Ajuste } \\
\cline { 2 - 8 } & $\chi^{2}$ & gl & GFI & NFI & CFI & RMSEA & AIC \\
\hline $\begin{array}{l}\text { Modelo sin } \\
\text { restricciones }\end{array}$ & $165.841^{*}$ & 74 & .932 & .944 & .968 & .055 & 281.841 \\
$\begin{array}{l}\text { Invarianza métrica } \\
176.713 *\end{array}$ & 81 & .928 & .940 & .966 & .054 & 278.713 \\
$\begin{array}{l}\text { Invarianza } \\
\text { factorial fuerte }\end{array}$ & $210.019 *$ & 91 & .916 & .929 & .958 & .056 & 292.019 \\
\hline $\begin{array}{l}\text { Nota: * p <.05; GFI = índice de bondad de ajuste; NFI = índice de ajuste } \\
\text { normado; CFI = índice de ajuste comparativo; RMSEA = error cuadrático } \\
\text { medio de aproximación; AIC = criterio de Información de Akaike }\end{array}$
\end{tabular}

La mayoría de los factores obtenidos en los análisis factoriales confirmatorios alcanzaron valores de consistencia interna por encima de .70 en ambas muestras (mujeres y hombres) (Tabla 8). En cuanto a la varianza media extraída todos los factores obtienen valores por encima de .50 .

Tabla 8

Coeficiente omega y alfa para el factor obtenido.

\begin{tabular}{lcccccc} 
& \multicolumn{3}{c}{ Mujeres } & \multicolumn{3}{c}{ Hombres } \\
\cline { 2 - 7 } \multicolumn{1}{c}{ Factor } & $\Omega(\mathrm{FC})$ & $\alpha$ & AVE & $\Omega(\mathrm{FC})$ & $\alpha$ & AVE \\
\cline { 2 - 7 } $\begin{array}{l}\text { Imagen Corporal / } \\
\begin{array}{l}\text { Ansiedad física } \\
\text { social }\end{array}\end{array}$ & .876 & .796 & .639 & .907 & .845 & .709 \\
$\begin{array}{l}\text { Fatiga / Pereza } \\
\text { Obligaciones / Falta } \\
\text { de tiempo }\end{array}$ & .793 & .792 & .657 & .720 & .717 & .563 \\
$\begin{array}{l}\text { Ambiente / } \\
\text { Instalaciones }\end{array}$ & .700 & .664 & .550 & .759 & .749 & .613 \\
\hline
\end{tabular}

Nota: $\mathrm{FC}=$ fiabilidad compuesta $\mathrm{AVE}=$ varianza media extraída

\section{Contraste de las medias entre mujeres y hombres}

Una vez comprobada la invarianza factorial, las diferencias entre las medias del factor de los dos grupos se estimaron tomando como referente la muestra de mujeres, fijando en 0 el valor de las medias para dicha muestra y estimando libremente el valor de las medias para la muestra de hombres. Las restricciones sobre los coeficientes de regresión e interceptos, requeridos para los contrastes entre las medias se realizaron automáticamente mediante el software AMOS 21 (Arbuckle, 2012). No se encontraron diferencias estadísticamente significativas entre hombres y mujeres deportistas en relación a los cuatro factores estudiados.

\section{DISCUSIÓN Y CONCLUSIONES}

El objetivo del presente estudio instrumental fue examinar la composición e invarianza factorial por género del ABPEF-M propuesta por RodríguezVillalobos et al. (2017) en deportistas universitarios mexicanos. Además de la estructura factorial de un instrumento, es necesario evaluar si la misma estructura factorial es aplicable a distintas poblaciones como mujeres y hombres (Abalo et al., 2006). En este sentido, los resultados del presente estudio mostraron que la estructura de cuatro factores (es decir, imagen corporal/ ansiedad física social, fatiga/ pereza, obligaciones/ falta de tiempo, y ambiente/ instalaciones) es fiable y válida tanto para la muestra total como para los hombres y mujeres separadamente.

Los análisis factoriales confirmatorios realizados en la muestra total apoyan la estructura factorial de cuatro factores (imagen corporal/ ansiedad física social, fatiga/ pereza, obligaciones/ falta de tiempo, y ambiente/ instalaciones). Los factores obtenidos presentaron saturaciones factoriales estandarizadas adecuadas, los cuales, en general, se corresponden con la estructura propuesta para el cuestionario por Rodríguez-Villalobos et al. (2017). No obstante, cuando se eliminaron dos de los 13 ítems (es decir, 5. "No estar "en forma" para practicar ejercicio", y 12. "Sentir vergüenza porque me están mirando mientras hago ejercicio") el modelo fue significativamente mejor. Además, los factores correlacionaban entre sí de forma positiva y estadísticamente significativa, indicando que a medida que aumentaba la percepción de barreras en alguno de ellos también aumenta en los otros.

$\mathrm{El}$ análisis factorial confirmatorio tanto en la muestra de mujeres como de hombres indicó que el ajuste de los datos al modelo teórico de 11 ítems agrupados en cuatro factores fue óptimo. Además, los factores así obtenidos presentaron saturaciones factoriales estandarizadas adecuadas. Por otro lado, los factores en ambas muestras también evidenciaron una consistencia interna aceptable, particularmente si se considera el número reducido de ítems en cada uno de ellos (Revelle y Zinbarg, 2009).

Por lo anteriormente dicho, los resultados del análisis de la invarianza factorial entre mujeres y hombres indicaron una alta congruencia entre pares de factores. Esto sugiere la existencia de fuerte evidencia de la 


\section{Mondaca, Zueck, Mayorga-Vega, Flores, Benitez, Peinado}

validación cruzada de la medida y, por tanto, de la estabilidad de la estructura (Abalo et al., 2006). Por otro lado, las comparaciones entre los grupos no reflejaron diferencias estadísticamente significativas entre los deportistas universitarios hombres y mujeres. No se encontraron estudios previos con el mismo instrumento que en el presente estudio, así como que los hallazgos sobre el tema en la literatura previa son contradictorios. Por ejemplo, en línea con el presente estudio, Rodríguez-Castellanos et al. (2017) en una muestra de estudiantes universitarios no encontraron diferencias estadísticamente significativas entre hombres y mujeres en las barreras percibidas por influencias sociales (similar a imagen corporal/ ansiedad físico social del ABPEF-M), falta de tiempo (similar a falta de tiempo/ obligaciones del ABPEF$\mathrm{M})$, falta de energía y falta de voluntad (similar a fatiga/ pereza del ABPEF-M), y falta de recursos (similar a ambiente/ instalaciones del ABPEF-M), así como otras dimensiones no recogidas por el ABPEF$\mathrm{M}$ tales como temor a las lesiones. Excepcionalmente, tan solo las mujeres presentaron mayores barreras por falta de habilidad para la realización de AF que los hombres, la cual no está presente en el ABPEF-M utilizado en este estudio. Asimismo, ColmenaresPedraza y Herrera-Medina (2018) en una muestra de adultos jóvenes y mayores no encontraron ninguna asociación estadísticamente significativa entre las barreras para la práctica de AF y el género. No obstante, en los estudios anteriores no se tuvieron en cuenta los hábitos de AF de los participantes. En este sentido, Sevil et al. (2017) en una muestra de estudiantes universitarios analizaron las diferencias por género y niveles de AF. Estos autores encontraron que tanto las mujeres que cumplían como las que no cumplían con las recomendaciones de práctica de $\mathrm{AF}$ percibían estadísticamente mayores barreras contextuales y de seguridad en la práctica (similar a ambiente/ instalaciones del ABPEF-M), referidas a tiempo (similar a Falta de tiempo/ Obligaciones del ABPEF-M) y referidas a la capacidad de disfrute (similar en algunos de sus ítems a Fatiga/ Pereza del ABPEF-M) que los hombres de su mismo nivel de AF.

En cuanto a las limitaciones del presente estudio, primero encontramos que los participantes fueron estudiantes universitarios deportistas, lo que limita la posibilidad de generalizar los hallazgos del presente estudio a otras poblaciones. Estudios futuros deberían ampliar la muestra, por ejemplo, agregando adultos jóvenes que no sean estudiantes universitarios y/ o jóvenes y adultos mayores. La segunda limitación proviene del propio instrumento de medición, que se basa en el autoreportaje y que por ello podría contener los sesgos que se derivan de la deseabilidad social. Estudios futuros también deberían comprobar si el cuestionario resulta útil para explicar la falta de motivación y de adherencia hacia el inicio y el mantenimiento de una conducta activa.

En conclusión, el análisis de las propiedades psicométricas del cuestionario ABPEF-M ha mostrado que la estructura tetrafactorial es viable, adecuada e invariante entre mujeres y hombres deportistas mexicanos universitarios. Atendiendo a criterios estadísticos y sustantivos, la estructura de cuatro factores ha mostrado adecuados indicadores de ajuste, fiabilidad y validez. Estudio futuros con diversas muestras son necesarios con el fin de contrastar los hallazgos obtenidos en la presente investigación.

\section{APLICACIONES PRÁCTICAS}

Disminuir la percepción de barreras para realizar ejercicio físico, por ejemplo como lo sugiere Sevil et al. (2017), desarrollando programas y estrategias de intervención, que permitan mejorar la gestión del tiempo de ocio y la motivación de los estudiantes universitarios para reducir las barreras de práctica de AF y asentar estilos de vida más activos y autónomos en esta etapa, sin duda contribuye a abatir el sedentarismo y con ello a la mejora de la salud de las personas, de ahí la importancia de contar con instrumentos válidos y confiables para su medición. Por ello, el presente estudio analiza la composición e invarianza factorial por género del ABPEF-M en deportistas universitarios mexicanos. Este estudio además sirve de referencia para futuras investigaciones sobre el estudio de instrumentos para la medición de la percepción de barreras relacionadas con la práctica del ejercicio físico en poblaciones con diferentes factores personales y culturales. Finalmente, el presente instrumento será de gran utilidad para la aplicación en diferentes ámbitos como, por ejemplo, estudios descriptivos o de intervención.

\section{AGRADECIMIENTOS}

La Secretaría de Educación Pública-Subsecretaría de Educación Superior-Dirección de Superación Académica- Programa para el Desarrollo Profesional Docente (DE-13 -6894) quién financió este estudio. 


\section{Composición e Invarianza factorial del cuestionario ABPEF-M}

Así como la facilidad para la financiación de la publicación (CONACYT, Redes Temáticas, No. 269614).

\section{REFERENCIAS}

1. Abalo, J., Lévy, J., Rial, A. y Varela, J. (2006). Invarianza factorial con muestras múltiples. En J. Lévy (Ed.), Modelización con Estructuras de Covarianzas en Ciencias Sociales (pp. 259-278). Madrid: Netbiblo.

2. Arbuckle, J. R. (2012). AMOS users guide version 21.0. Chicago, IL: Marketing Department, SPSS Incorporated.

3. Ato, M., López, J. J. y Benavente, A. (2013). Un sistema de clasificación de los diseños de investigación en psicología. Anales de Psicología, 29(3), 1038-1059.

4. Byrne, B. M. (2016). Structural Equation Modeling With AMOS: Basic Concepts, Applications, and Programming. New York, NY: Routledge.

5. Capdevila, L. (2005). Actividad física y estilo de vida saludable. Girona: Documenta Universitaria.

6. Colmenares-Pedraza, J. A. y Herrera-Medina, R. (2018). Prevalencia de actividad física y beneficios y barreras en trabajadores de Villavicencio, Colombia. Revista Salud UIS, 50(1), 37-45. doi: 10.18273/revsal.v50n12018004

7. Cheung, G. W. y Rensvold, R. B. (2002). Evaluating goodness-of-fit indexes for testing measurement invariance. Structural Equation Modeling, 9(2), 233-255. doi: 10.1207/s15328007SEM0902_5

8. Elosua, P. y Zumbo, B. D. (2008). Coeficientes de fiabilidad para escalas de respuesta categórica ordenadas. Psicothema, 20(4), 896-901.

9. Fornell, C., y Larcker, D. F. (1981). Evaluating structural equations models with unobservable variables and measurement error. Journal of Marketing Research, 18(1), 39-50.

10. Gelabert, E., García-Esteve, L., Martín-Santos, R., Gutiérrez, F., Torres, A. y Subirà, S. (2011). Psychometric properties of the Spanish version of the Frost Multidimensional Perfectionism Scale in women. Psicothema, 23(1), 133-139.
11. Gunnell, K. E., Brunet, J., Wing, E. K. y Bélanger, M. (2015). Measuring Perceived Barriers to Physical Activity in Adolescents. Pediatric Exercise Science, 27(2), 252-261. doi: 10.1123/pes.2014-0067

12. Guthold, R., Stevens, G. A., Riley, L. M. y Bull, F. C. (2018). Worldwide trends in insufficient physical activity from 2001 to 2016: a pooled analysis of 358 population-based surveys with 1.9 million participants. The Lancet Global Health, $6(10), \quad 1077-1086$. doi: 10.1016/S2214109X(18)30357-7

13. Huai, P., Xun, H., Reilly, K. H., Wang, Y., Ma, W. y Xi, B. (2013). Physical activity and risk of hypertension: a meta-analysis of prospective cohort studies. Hypertension, 62(6), 1021-1026. doi: 10.1161/HYPERTENSIONAHA.113.01965

14. Li, J. y Siegrist, J. (2012). Physical activity and risk of cardiovascular disease -a meta-analysis of prospective cohort studies. International Journal of Environmental Research and Public Health, 9(2), 391-407. doi: 10.3390/ijerph9020391

15. Murgui, S., García, C. y García, Á. (2016). Efecto de la práctica deportiva en la relación entre las habilidades motoras, el autoconcepto físico y el autoconcepto multidimensional. Revista de Psicología del Deporte, 25(1), 19-25.

16. Niñerola, J., Capdevila, L. y Pintanel, M. (2006). Barreras percibidas y actividad física: el autoinforme de barreras para la práctica de ejercicio físico. Revista de Psicología del Deporte, 15(1), 53-69.

17. Nunnally, J. C. y Bernstein, I. H. (1995). Teoría Psicométrica. México: McGraw-Hill.

18. Poitras, V. J., Gray, C. E., Borghese, M. M., Carson, V., Chaput, J.-P., Janssen, I., . . . Tremblay, M. S. (2016). Systematic review of the relationships between objectively measured physical activity and health indicators in schoolaged children and youth. Applied Physiology, Nutrition, and Metabolism, 41(6), 197-239. doi: 10.1139/apnm-2015-0663

19. Requena-Pérez, C. M., Martín-Cuadrado, A. M. y Lago-Marín, B. S. (2015). Imagen corporal, autoestima, motivación y rendimiento en practicantes de danza. Revista de Psicología del Deporte, 24(1), 37-44. 


\section{Mondaca, Zueck, Mayorga-Vega, Flores, Benitez, Peinado}

20. Revelle, W. y Zinbarg, R. E. (2009). Coefficients alpha, beta, omega and the glb: comments on Sijtsma. Psychometrika, 74(1), 145-154. doi: 10.1007/s11336-008-9102-z

21. Rodríguez-Castellanos, F. A., Valencia, S. C., Gaitán-Guzmán, E. M., González-Rodríguez, S. A. y León-Díaz, J. D. (2017). Hábitos saludables, motivos y barreras en la realización de actividad física en estudiantes universitarios. Revista de Investigación: Cuerpo, Cultura y Movimiento, 7(1), 81-102.

22. Rodríguez-Villalobos, J., Viciana, J., Tristán, J. L. y Blanco, J. R. (2017). Composición factorial del cuestionario ABPEF en universitarios mexicanos. Revista de Psicología del Deporte, 26(2), 43-50.

23. Rodríguez, M. N. y Ruiz, M. A. (2008). Atenuación de la asimetría y de la curtosis de las puntuaciones observadas mediante transformaciones de variables: Incidencia sobre la estructura factorial. Psicológica, 29, 205-227.

24. Samperio, J., Jiménez-Castuera, R., Lobato, S., Leyton, M. y Claver, F. (2016). Variables motivacionales predictoras de las barreras para la práctica de ejercicio físico en adolescentes. Cuadernos de Psicología del Deporte, 16(2), 6576.

25. Sevil, J., Práxedes, A., Zaragoza, J., Del Villar, F. y García-González, L. (2017). Barreras percibidas para la práctica de actividad física en estudiantes universitarios. Diferencias por género y niveles de actividad física. Universitas Psychologica, 16(4), 1-15. doi: 10.11144/Javeriana.upsy16-4.bppa

26. Sigal, R. J., Kenny, G. P., Wasserman, D. H., Castaneda-Sceppa, C. y White, R. D. (2006). Physical activity/exercise and type 2 diabetes: a consensus statement from the American Diabetes Association. Diabetes Care, 29(6), 1433-1438. doi: 10.2337/dc06-9910

27. Sijtsma, K. (2009). On the use, the misuse, and the very limited usefulness of Cronbach's alpha. Psychometrika, 74(1), 107-120. doi: 10.1007/s11336-008-9101-0

28. Thompson, B. (2004). Exploratory and Confirmatory Factor Analysis. Understanding concepts and applications. . Washington, D C: American Psychological Association.
29. Urrutia, S., Azpillaga, I., de Cos, G. L. y Muñoz, D. (2010). Relación entre la percepción de estado de salud con la práctica físicodeportiva y la imagen corporal en adolescentes. Cuadernos de Psicología del Deporte, 20 (2), 51-56.

30. World Health Organization. (2014). Global status report on noncommunicable diseases 2014. Switzerland: World Health Organization.

31. World Health Organization. (2018). Global action plan on physical activity 2018-2030: more active people for a healthier world. Switzerland: World Health Organization.

32. Zhong, S., Ma, T., Chen, L., Chen, W., Lv, M., Zhang, X. y Zhao, J. (2016). Physical Activity and Risk of Lung Cancer: A Meta-analysis. Clinical Journal of Sport Medicine, 26(3), 173-181. doi: 10.1097/JSM.0000000000000219 